University of Nebraska - Lincoln

DigitalCommons@University of Nebraska - Lincoln

$9-1-1998$

\title{
Can photoemission accurately probe the bulk electronic structure of the complex oxides?
}

Jaewu Choi

University of Nebraska-Lincoln, jchoi@ece.eng.wayne.edu

C. Waldfried

University of Nebraska-Lincoln

Sy_Hwang Liou

University of Nebraska-Lincoln, sliou@unl.edu

Peter A. Dowben

University of Nebraska-Lincoln, pdowben@unl.edu

Follow this and additional works at: https://digitalcommons.unl.edu/physicsdowben

Part of the Physics Commons

Choi, Jaewu; Waldfried, C.; Liou, Sy_Hwang; and Dowben, Peter A., "Can photoemission accurately probe the bulk electronic structure of the complex oxides?" (1998). Peter Dowben Publications. 119.

https://digitalcommons.unl.edu/physicsdowben/119

This Article is brought to you for free and open access by the Research Papers in Physics and Astronomy at DigitalCommons@University of Nebraska - Lincoln. It has been accepted for inclusion in Peter Dowben Publications by an authorized administrator of DigitalCommons@University of Nebraska - Lincoln. 


\title{
Can photoemission accurately probe the bulk electronic structure of the complex oxides?
}

\author{
Jaewu Choi, C. Waldfried, S.-H. Liou, and P. A. Dowben ${ }^{\text {a) }}$ \\ Department of Physics and Astronomy and the Center for Materials Research and Analysis, \\ Behlen Laboratory of Physics, University of Nebraska-Lincoln, Lincoln, Nebraska 68588-0111
}

(Received 29 September 1997; accepted 4 May 1998)

\begin{abstract}
The surface of the complex oxides, in particular the manganese perovskites, is dominated by a different free enthalpy at the surface. This can be demonstrated by surface segregation after annealing the samples. By comparing relative $\mathrm{x}$-ray photoelectron spectroscopy intensities of the manganese perovskite $\mathrm{La}_{1-x} \mathrm{Ca}_{x} \mathrm{MnO}_{3}(x=0.1,0.35)$ for different emission angles, the profile of elemental concentration was determined. Analysis shows evidence for pronounced surface segregation of calcium. Our results suggest that there are strong differences between surface and bulk enthalpies without segregation or surface relaxation. This is consistent with the previously observed surface resonance in the electronic band structure. (c) 1998 American Vacuum Society. [S0734-2101(98)00505-3]
\end{abstract}

\section{INTRODUCTION}

Recent angle resolved photoemission and inverse photoemission studies of the manganese perovskites $\left(\mathrm{La}_{0.65} \mathrm{~A}_{0.35} \mathrm{MnO}_{3}, \mathrm{~A}=\mathrm{Ca}, \mathrm{Ba}\right)$ provide strong evidence for the existence of surface resonances in the experimental band structure. $^{1,2}$ These results lent support to the theoretical postulate, by Liu and Klemm, ${ }^{3}$ and others ${ }^{4}$ that the complex oxides exhibit a distinct surface electronic structure. The high temperature superconducting oxides could support surface electronic states from the terminal layer structure. ${ }^{3,4}$ There is no reason, a priori, to suppose that in the complex oxides the surface terminal layer must be representative of the bulk even if the sample is perfectly cleaved to expose a surface with bulk structure and composition. Furthermore, even if the terminal layer of the perovskites is compositionally and chemically identical with the bulk, there is also no reason to suppose that the surface free enthalpy is identical to the bulk. Differences in the surface free enthalpy, apart from any compositional or chemical differences from the bulk material, would be expected to lead to differences in the surface electronic structure as compared to the bulk.

Because the surface free energy is expected to be different from the bulk, surface segregation is expected. Surface segregation in ionic solids must be considered because of the existence of the space charge layer in the near surface region, providing a strong chemical potential. The bonding character of these transition metal oxides, $\mathrm{La}_{1-x} \mathrm{~A}_{x} \mathrm{MnO}_{3}(\mathrm{~A}=\mathrm{Ca}, \mathrm{Ba}$, $\mathrm{Sr}$ ) is mainly ionic. The surface segregation has been observed in many oxide alloys, including the surface segregation of calcium. ${ }^{5-7}$ Recently we have shown that the extent of surface segregation, ${ }^{8}$ as well as the surface composition of the terminal layer ${ }^{8,9}$ depend upon the calcium doping level in $\mathrm{La}_{1-x} \mathrm{Ca}_{x} \mathrm{MnO}_{3}$. In this article, we wish to make the point that this has important consequences for the experimentalist attempting to measure the electronic structure of the bulk using photoemission, even in the absence of any segregation

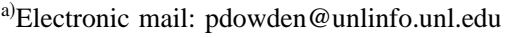

whatsoever. If one is able to prepare a surface with an ideal termination of the bulk lattice we would expect to observe a surface electronic structure different from the bulk, similar to the predictions of theory. ${ }^{3,4}$

\section{SAMPLE PREPARATION AND EXPERIMENTAL DETAILS}

The $\mathrm{La}_{1-x} \mathrm{Ca}_{x} \mathrm{MnO}_{3}(x=0.1,0.35)$ thin films were grown on (100) $\mathrm{LaAlO}_{3}$ substrates by radio frequency (rf) sputtering in a 2:1 argon/oxygen atmosphere maintained at 20 mTorr. The bulk chemical composition of the films was determined from energy dispersive analysis by $\mathrm{x}$-ray (EDAX) of $\mathrm{x}$-ray emission spectroscopy (XES) and found to be similar to the targets with final compositions of $\mathrm{La}_{0.9} \mathrm{Ca}_{0.1} \mathrm{MnO}_{3}$ and $\mathrm{La}_{0.65} \mathrm{Ca}_{0.35} \mathrm{MnO}_{3}$. The thickness of the $\mathrm{La}_{0.9} \mathrm{Ca}_{0.1} \mathrm{MnO}_{3}$ and $\mathrm{La}_{0.65} \mathrm{Ca}_{0.35} \mathrm{MnO}_{3}$ samples was nominally $2500 \AA$. The films were subsequently annealed at $900{ }^{\circ} \mathrm{C}$ in an oxygen atmosphere of $2 \mathrm{~atm}$ for $10 \mathrm{~h}$ in order to improve the compositional homogeneity of the samples. The crystallinity and orientation were determined by $\mathrm{x}$-ray diffraction and later by low energy electron diffraction (LEED). Samples were cleaned by repeated annealing and exposure to low energy electrons to stimulate the desorption of contaminants as described elsewhere. ${ }^{1}$ Appropriate surface preparation was established by x-ray photoelectron spectroscopy (XPS), ultraviolet photoemission, and inverse photoemission. Prior to each experiment, samples were again cleaned by annealing at $760 \mathrm{~K}$ for $2 \mathrm{~h}$. Exposure of the samples to oxygen in the vacuum system was found not to affect any of the photoemission spectra or the LEED.

XPS was undertaken with the $\mathrm{Mg} K \alpha$ line $(1253.6 \mathrm{eV})$ from a PHI Model 04-548 dual anode x-ray source. Energy distribution curves of the elemental core levels were acquired with a large hemispherical electron energy analyzer (PHI Model 10-360 precision energy analyzer). The core level spectra were measured at two different pass energies: 11.75 and $23.5 \mathrm{eV}$. The results were independent of pass energy, and therefore resolution. The Fermi level was established 
from tantalum in electrical contact with the sample. The binding energy of core levels are reported with respect to this Fermi level. All the core level data reported herein were taken at room temperature. The ultrahigh vacuum (UHV) chamber used for core level photoemission was maintained at a vacuum of about $2 \times 10^{-10}$ Torr and was equipped with LEED, inverse photoemission, a helium lamp, and mass spectrometer as described elsewhere. ${ }^{1}$

The angle resolved photoemission spectroscopy experiments on $\mathrm{La}_{0.65} \mathrm{Ba}_{0.35} \mathrm{MnO}_{3}$ were performed using synchrotron radiation dispersed by a $3 \mathrm{~m}$ toroidal grating monochromator. These experiments were conducted at the Synchrotron Radiation Center in Stoughton, WI, and are discussed in detail elsewhere. ${ }^{1,2}$ The UHV chamber was maintained at a base pressure between 6 and $8 \times 10^{-11}$ Torr and was equipped with an angle resolved electron analyzer with an acceptance angle of $\pm 1^{\circ}$. The combined energy resolution of the analyzer and the light source was about $150 \mathrm{meV}$ or less. The chamber was also equipped with a LEED system for establishing long range surface crystallographic order. The work presented for $\mathrm{La}_{0.65} \mathrm{Ba}_{0.35} \mathrm{MnO}_{3}$ was undertaken with a light incidence angle of $65^{\circ}$ (p-polarized light). Throughout this work, the in-plane component of the vector potential $\mathbf{A}$, in the plane of the surface, is in the plane defined by the sample surface normal and the detector (even geometry), i.e., parallel to the $\bar{\Gamma}-\bar{X}$ direction.

\section{RESULTS}

Along the $c$ axis, parallel to the surface normal in our single crystal thin films, these transition metal oxides have a layer structure of two different layers: alternatively $\mathrm{Mn}-\mathrm{O}$ and $\mathrm{La}-\mathrm{Ca}-\mathrm{O}$ layers. Thus there are two possible surface or terminal layers. In order to determine the dominant terminal layer, the $\mathrm{La} 3 d$, Mn $2 p$, and $\mathrm{Ca} 2 p$ core level spectra from $\mathrm{La}_{0.9} \mathrm{Ca}_{0.1} \mathrm{MnO}_{3}$ and $\mathrm{La}_{0.65} \mathrm{Ca}_{0.35} \mathrm{MnO}_{3}$ were taken at room temperature as a function of the emission angle with respect to the surface normal, as described elsewhere, ${ }^{8-9}$ and shown in Fig. 1 (top). The photoelectron intensities from the $\mathrm{Mn} 2 p$, La $3 d$, and $\mathrm{Ca} 2 p$ core levels are normalized with each core level cross section ${ }^{10}$ and the data have been plotted as to eliminate any instrumental effects. The terminal layer has a large contribution to the intensity of the core level spectra because of the limited electron mean free path, and thus is an indication of the dominant terminal layer. A normalized ratio of unity would indicate that there is no dominant terminal layer: i.e., both $\mathrm{Mn}-\mathrm{O}$ and $\mathrm{La}-\mathrm{Ca}-\mathrm{O}$ are terminal layers in rough equal portions. This is not the case, ${ }^{8,9}$ as can be clearly seen in Fig. 1 (at top). The overall Mn $2 p_{1 / 2}$ and $2 p_{3 / 2}$ core level signals for the $\mathrm{La}_{0.65} \mathrm{Ca}_{0.35} \mathrm{MnO}_{3}$ are stronger relative to the summed and normalized calcium $2 p_{1 / 2}$ and $2 p_{3 / 2}$ plus lanthanum $3 d_{3 / 2}$ and $3 d_{5 / 2}$ core level signals than is the case for $\mathrm{La}_{0.9} \mathrm{Ca}_{0.1} \mathrm{MnO}_{3}$. Thus these differences in the $\mathrm{Mn}$ to $\mathrm{La}-\mathrm{Ca}$ core level intensities imply that the terminal layer at the surface is different for each calcium composition. The data suggest that the dominant oxide plane in the terminal layer of $\mathrm{La}_{0.65} \mathrm{Ca}_{0.35} \mathrm{MnO}_{3}$ film is the $\mathrm{Mn}-\mathrm{O}$

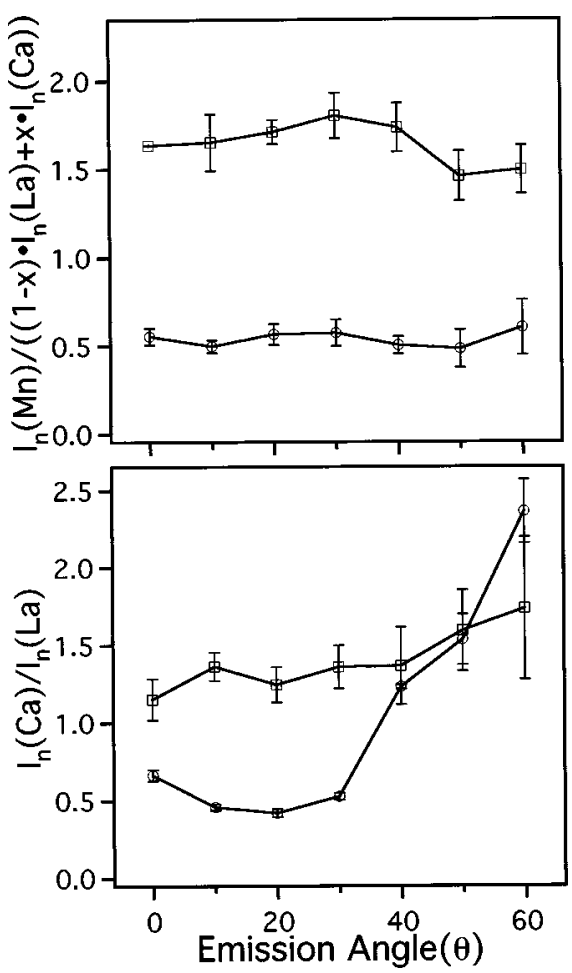

Fig. 1. Normalized core level intensity ratio of Mn $2 p$ to La $3 d$ plus Ca $2 p$ core levels as a function of a emission angle for the $\mathrm{La}_{0.9} \mathrm{Ca}_{0.1} \mathrm{MnO}_{3}(\mathrm{O})$ and $\mathrm{La}_{0.65} \mathrm{Ca}_{0.35} \mathrm{MnO}_{3}(\square)$ perovskites (top). The data has been corrected for cross section. Also shown is the $\mathrm{La}$ to $\mathrm{Ca}$ ratio as a function of emission angle for the $\mathrm{La}_{0.9} \mathrm{Ca}_{0.1} \mathrm{MnO}_{3}(\bigcirc)$ and $\mathrm{La}_{0.65} \mathrm{Ca}_{0.35} \mathrm{MnO}_{3}(\square)$ perovskites (bottom)

layer while the dominant oxide plane in the terminal layer of the $\mathrm{La}_{0.9} \mathrm{Ca}_{0.1} \mathrm{MnO}_{3}$ sample is the $\mathrm{La}-\mathrm{Ca}-\mathrm{O}$ layer.

One can make use of the limited electron mean free path to determine the segregation of one component preferentially to the surface and near surface region. ${ }^{11-13}$ Either different electron kinetic energies of characteristics electron spectroscopy signals (surface sensitive techniques like AES or XPS) or photoelectron emission angle dependence can be employed. The profile of the elemental concentrations can be determined by the intensity of the emitted core level photoelectron from each element and the electron mean free path. We have analyzed the elemental concentrations by the angle resolved $\mathrm{x}$-ray core level photoemission signals using a previously developed semiempirical analysis method. ${ }^{11,12}$

Apparent surface concentrations for a binary system $\left(A_{b} B_{1-b}\right)$ can be determined from the core level emission angle dependent intensity ration between the components of the alloy where the intensity is normalized by the cross sections $\left(\sigma_{A}, \sigma_{B}\right)$ of the core level of the each element $A$ and $B$. The normalized intensity ratio is given as:

$$
R(\theta)=\frac{I_{A}(\theta) / \sigma_{A}}{I_{B}(\theta) / \sigma_{B}},
$$

where $\theta$ is the emission angle with respect to the surface normal. From the normalized intensity ratio, the apparent surface concentration is given as: 


$$
C(\theta)=\frac{R(\theta)}{1+R(\theta)}
$$

A summation is undertaken to account for each layer contributing to the photoemission signal. Here, the atomic fraction of dopant $A$ at the $j$ th layer is written as:

$$
f_{j}=b+\delta \cdot e^{-j / G},
$$

where the parameters $b, \delta$ and $G$ are the bulk fraction for element $A$, The segregation at the topmost layer and the segregation depth in units of the distance $d$ between layers, respectively. Thus the normalized intensity ratio can be rewritten as:

$$
R(\theta)=\frac{\sum_{j=0}^{\infty} f_{j} e^{-j / \lambda_{A}}}{\sum_{j=0}^{\infty}\left(1-f_{j}\right) e^{-j / \lambda_{B}}},
$$

where $\lambda_{A}(\theta)$, and $\lambda_{B}(\theta)$ are the effective mean free paths (in units of distance $d$ between layers) for photoelectrons emitted from the particular core level, for component $A$ and $B$, respectively. An exponential profile is assumed because this is the most reasonable expectation for the variation of the free energy from the surface to the bulk..$^{11,12}$

In the analysis of the XPS results, we need to determine the media absorbing the photoelectrons. For the manganese perovskites, the mean free path of photoelectrons is dominated by the $\mathrm{Mn}-\mathrm{O}$ layers. The mean free path of the electrons in these materials were adapted from the calculated mean free paths of Penn. ${ }^{14}$ We have adopted a mean free path of $6.5 \AA$ for the lanthanum core levels and $12 \AA$ for the calcium core levels. The differences in the mean free paths between the calcium core levels and the lanthanum core levels are a result of the electron kinetic energies of the pertinent from Ca $2 p$ (897-902 eV) and La $3 d$ (393-416 eV) core levels. Scofield's results for kinetic energy dependent cross sections using $\mathrm{Mg} K \alpha$ line $(1253.6 \mathrm{eV})$ were used here. ${ }^{10}$

In addition, as the terminal layer is different for each calcium composition, $x=0.1$ and $x=0.35$, we have accounted for the need to begin the summation [Eq. (4)] with a different terminal layer. We applied the analysis of Eqs. (1)-(4), assuming the terminal layer for $\mathrm{La}_{0.9} \mathrm{Ca}_{0.1} \mathrm{MnO}_{3}$ is the $\mathrm{La}-$ $\mathrm{Ca}-\mathrm{O}$ layer while for $\mathrm{La}_{0.65} \mathrm{Ca}_{0.35} \mathrm{MnO}_{3}$, the terminal layer is the $\mathrm{Mn}-\mathrm{O}$ layer.

For $\mathrm{La}_{1-x} \mathrm{Ca}_{x} \mathrm{MnO}_{3}$, the summation, with respect to the layers that contribute to the signal in Eq. (4), is done over $2 n$ for $\mathrm{Ca}$ and $\mathrm{La}$, instead of every layer where $n$ is a positive integer and includes 0 (the surface). We must account for the superlattice layered structure for $\mathrm{La}_{1-x} \mathrm{Ca}_{x} \mathrm{MnO}_{3}$ with recurring $\mathrm{La}-\mathrm{Ca}-\mathrm{O}, \mathrm{Mn}-\mathrm{O}, \mathrm{La}-\mathrm{Ca}-\mathrm{O}$ and $\mathrm{Mn}-\mathrm{O}$ layers. Since we sum over every $2 n$ layers, we have begun integrating using a $\mathrm{La}-\mathrm{Ca}-\mathrm{O}$ terminal layer for $x=0.1$ and $\mathrm{Mn}-\mathrm{O}$ as a terminal layer for $x=0.35$. This analysis was applied to our XPS data for various emission angles as:
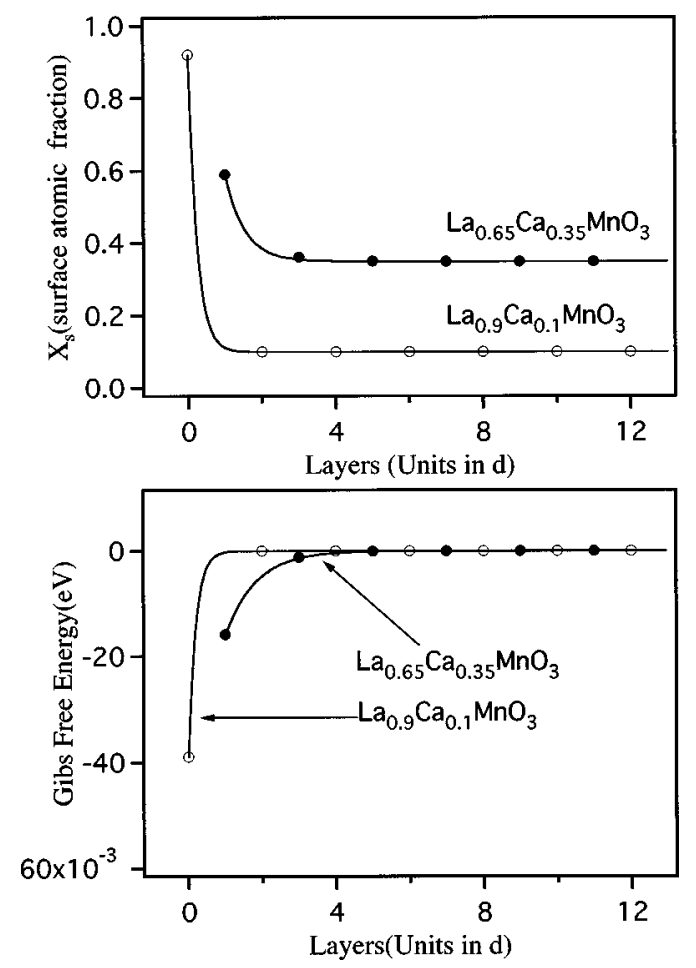

FIG. 2. Ca atomic fraction as a function of depth, in unit of $d$ ( $d$ $=1.96 \AA$ ) for both the $\mathrm{La}_{0.9} \mathrm{Ca}_{0.1} \mathrm{MnO}_{3}(\bigcirc)$ and $\mathrm{La}_{0.65} \mathrm{Ca}_{0.35} \mathrm{MnO}_{3}(\bullet)$ perovskites (at top). The $\mathrm{La}-\mathrm{Ca}-\mathrm{O}$ layers are the even layers for $\mathrm{La}_{0.9} \mathrm{Ca}_{0.1} \mathrm{MnO}_{3}$, beginning with the terminal layer $(d=0)$, while for the $\mathrm{La}_{0.65} \mathrm{Ca}_{0.35} \mathrm{MnO}_{3}$ sample, the $\mathrm{La}-\mathrm{Ca}-\mathrm{O}$ layers are the odd numbered layers beginning with the layer immediately below the surface $(d=1)$. The calculated difference in free enthalpy in both the $\mathrm{La}_{0.9} \mathrm{Ca}_{0.1} \mathrm{MnO}_{3}(O)$ and $\mathrm{La}_{0.65} \mathrm{Ca}_{0.35} \mathrm{MnO}_{3}(\bullet)$ perovskites as derived from the surface segregation (see text) are shown at the bottom.

$$
R(\theta)=\frac{\frac{b}{1-X}+\frac{\delta}{1-X \cdot Z}}{\frac{1-b}{1-Y}-\frac{\delta}{1-Y \cdot Z}},
$$

where $X=\exp \left[-2 / \lambda_{\mathrm{Ca}}(\theta)\right], \quad Y=\exp \left[-2 / \lambda_{\mathrm{La}}(\theta)\right]$ and $Z$ $=\exp (-2 / G)$.

The normalized intensity ratio has a very strong angular dependence. Fitting the experimental results for $\mathrm{La}_{0.9} \mathrm{Ca}_{0.1} \mathrm{MnO}_{3}$ with Eq. (5) provides a $\delta=0.82$ and a segregation depth of $G=0.4$ layers. The normalized intensity ratio of the photoelectron emitted from the $\mathrm{La}$ and $\mathrm{Ca}$ core levels for the $\mathrm{La}_{0.65} \mathrm{Ca}_{0.35} \mathrm{MnO}_{3}$ sample in contrast shows a smaller emission angle dependence. The fitting of the experimental results for $\mathrm{La}_{0.65} \mathrm{Ca}_{0.35} \mathrm{MnO}_{3}$ with Eq. (5) is excellent with a small surface segregation $(\delta=0.24)$ but a larger segregation depth $(G=0.9$ layers). This means that the surface segregation is much smaller for $\mathrm{La}_{0.65} \mathrm{Ca}_{0.35} \mathrm{MnO}_{3}$ than for $\mathrm{La}_{0.9} \mathrm{Ca}_{0.1} \mathrm{MnO}_{3}$ but the decay of the segregation from the surface into the bulk is longer. As a result of this fitting to the experimental normalized intensity ratio, shown in Fig. 1 (bottom), the $\mathrm{La}$ to $\mathrm{Ca}$ atomic fraction can now be plotted as a function of depth in units of layer $d(d=1.96 \AA)$, using Eq. (3) as shown in Fig. 2 (top). We see that as indicated by simple inspection of the data, there is a huge surface segre- 


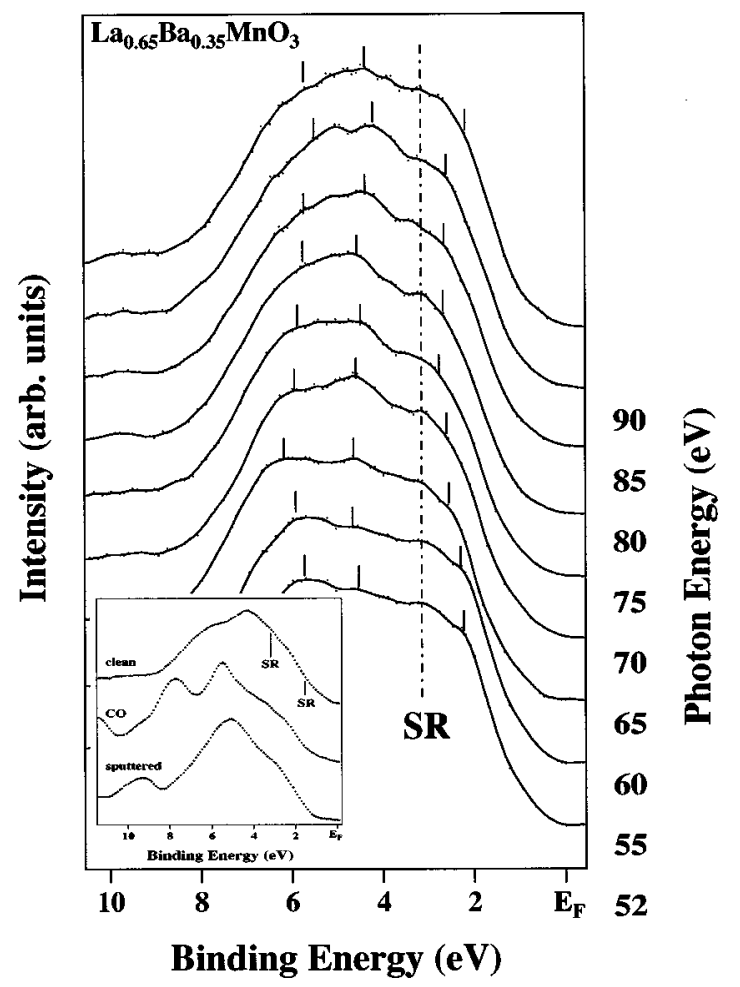

FIG. 3. Normal emission valence-band spectra of $\mathrm{La}_{0.65} \mathrm{Ba}_{0.35} \mathrm{MnO}_{3}$ acquired at $300 \mathrm{~K}$ for various photon energies. The insert shows the valence band spectra of a clean surface, a $\mathrm{CO}$ adsorbed surface and a sputtered surface of a thin film of $\mathrm{La}_{0.65} \mathrm{Ba}_{0.35} \mathrm{MnO}_{3}$. The three spectra were collected at normal emission with $p$-polarized light and a photon energy of $45 \mathrm{eV}$. SR marks the surface resonances.

gation of $\mathrm{Ca}$ for $x=0.1$, while for $x=0.35$, the surface segregation of $\mathrm{Ca}$ is significant, but much smaller.

In manganese perovskites samples, prepared with less surface segregation, there is evidence of a surface electronic structure. $^{1,2}$ The density of states near the Fermi level, in the manganese perovskites, is very sensitive to surface contamination and surface order. The clean photoemission spectrum of $\mathrm{La}_{0.65} \mathrm{Ba}_{0.35} \mathrm{MnO}_{3}$ is displayed in the insert to Fig. 3. The surface of $\mathrm{La}_{0.65} \mathrm{Ca}_{0.35} \mathrm{MnO}_{3}$ is fairly inert and is not prone to surface contamination, as determined by XPS and angle resolved ultraviolet photoemission spectroscopy (ARUPS). In contrast, the surface of $\mathrm{La}_{0.65} \mathrm{Ba}_{0.35} \mathrm{MnO}_{3}$ was found to be much more reactive. The middle photoemission spectrum in the insert to Fig. 3 is a $\mathrm{La}_{0.65} \mathrm{Ba}_{0.35} \mathrm{MnO}_{3}$ surface with adsorbed $\mathrm{CO}$. The three features in the valence band have been identified as the $5 \sigma(6 \mathrm{eV})$, the $1 \pi(8 \mathrm{eV})$, and the $4 \sigma(12 \mathrm{eV}$, partially displayed in the insert to Fig. 3) molecular orbitals of CO. ${ }^{1,2}$ The sputtered surface (the inset to Fig. 3) demonstrates that surface damage (defects) will greatly reduce the density of states and the concomitant photoemission signal near the Fermi level, i.e., in the region between 0 and $2 \mathrm{eV}$ binding energies. Both the suppression and the introduction of new states is observed with sputtering. An examination of the sputtered valence band spectrum in the insert to Fig. 3 reveals the strong suppression of the density of states from the Fermi level down to approximately $1.75 \mathrm{eV}$ binding energy. The intensity of a state at approximately $3.35 \mathrm{eV}$ also decreases with sputtering. The intensity of these same states were also seen to decrease with $\mathrm{CO}$ adsorption. There is a similar suppression of states just above the Fermi energy seen in inverse photoemission. ${ }^{1}$

While some of the valence band states are suppressed with sputtering, others are seen to increase in intensity and dominate the valence spectrum. The intensity of the oxygen $2 p$ derived state at $6 \mathrm{eV}^{1,2}$ increases with sputtering and dominates the valence band and a new state at $10 \mathrm{eV}$ is also observed. In addition to the above modifications of the valence band of $\mathrm{La}_{0.65} \mathrm{Ba}_{0.35} \mathrm{MnO}_{3}$ with sputtering, the disordered surface exhibited little or no dispersion.

The suppression of the $\mathrm{La}_{0.65} \mathrm{Ba}_{0.35} \mathrm{MnO}_{3} t_{2 g}$ band at 3.35 eV with CO contamination and disorder (Fig. 3) supports our premise that this state is localized at the surface-vacuum interface. While the band at $1.27 \mathrm{eV}$ does appear to be localized at the surface-vacuum interface, we refrain from identifying this band as a surface resonance until further information can be obtained.

Unlike other photoemission features, the state at $3.35 \mathrm{eV}$ does not disperse in binding energy with photon energy (wave vector normal to the surface in normal emission), as seen in Fig. 3. ${ }^{1,2}$ This two dimensionality of state combined with surface sensitivity is consistent with considerable oscillator strength at the surface. The $3.35 \mathrm{eV}$ state cannot be considered a surface state because it does not reside in a gap of the projected bulk bands ${ }^{2}$ and therefore must be defined as a surface resonance. Additionally, the increased intensity of this state with emission angle in Fig. 3 is also characteristic of surface localization. ${ }^{1,2}$ Taken together, these results conclusively identify the $t_{2 g}$ band at $3.35 \mathrm{eV}$, and possibly the $e_{g}$ band at $1.27 \mathrm{eV}$, as surface resonances.

\section{DISCUSSION}

The XPS results for the $\mathrm{La}_{0.65} \mathrm{Ca}_{0.35} \mathrm{MnO}_{3}$ sample are consistent with the assignment of the terminal layer based upon valence band photoemission. ${ }^{1,2}$ The existence of dominant terminal layers, rather than termination of the crystal in a random distribution of different layers, has also been observed in angle resolved XPS studies of other complex oxides: $\mathrm{YBa}_{2} \mathrm{Cu}_{3} \mathrm{O}_{7-x}{ }^{15}$ and $\mathrm{La}_{1-x} \mathrm{Sr}_{x} \mathrm{MnO}_{3}$. ${ }^{16}$ The segregation of calcium in oxides should also be expected. ${ }^{4-6}$ The extent of the calcium segregation is a strong indication that the surface enthalpy differs significantly from the bulk.

Surface segregation can be related to the enthalpy by a standard statistical model. In the statistical-mechanical model of segregation, ${ }^{17}$ the total free energy $F$ for the system consisting of several elements is written as:

$$
F=\sum_{i} n_{i}^{b} g_{i}^{b}+n_{i}^{s} g_{i}^{s}-k_{B} T \ln \Omega,
$$

where $n_{i}^{b}$ and $n_{i}^{s}$ are the number of bulk and surface atoms of type $i$ with individual free energies $g_{i}^{b}$ and $g_{i}^{s}$, respectively.

For a two component system (an oversimplification to the $\mathrm{La}-\mathrm{Ca}-\mathrm{O}$ layers admittedly), an Arrhenius expression is obtained: 


$$
n_{1}^{s} / n_{2}^{s}=n_{1} / n_{2} \exp \left(-H / k_{B} T\right),
$$

where $H$ is the enthalpy of segregation.

Equation (6) implies that the surface segregation is a competition to minimize the total free energy by a maximization of entropy by evenly mixing two elements and a minimization of free energy of each element. From Eq. (7), the enthalpy can be determined as a function of the atomic fraction. Based upon the large calcium segregation, we therefore find that the free enthalpy, at the surface of the $\mathrm{La}_{0.9} \mathrm{Ca}_{0.1} \mathrm{MnO}_{3}$ sample, is substantially different from the bulk as indicated in Fig. 2 (bottom). The differences in the free enthalpy of the $\mathrm{La}_{0.65} \mathrm{Ca}_{0.35} \mathrm{MnO}_{3}$ sample are not as significant as for the $\mathrm{La}_{0.9} \mathrm{Ca}_{0.1} \mathrm{MnO}_{3}$ sample. Corrections for surface relaxation and surface strain have not been included in this analysis and would lead to further increases in the difference in the surface enthalpy. The surface Gibbs free energy difference extracted from the experimental segregation data using a very simple model (about 50-150 meV) is smaller than the energy difference between the gap observed in photoemission ${ }^{18}$ and tunneling spectroscopy ${ }^{19}$ of more than $200 \mathrm{meV}$ and what is expected based upon the transition temperature in the manganese perovskites $(10-30 \mathrm{meV})$. This energy difference may well be part of the explanation for the differences in the Fermi surface from angle resolved photoemission and de Haas-van Alphen measurements. ${ }^{20}$ A more sophisticated analysis that includes surface relation, surface reconstructions and surface strain is likely to provide an energy that bridges these differences far better.

We are left with several conclusions. First, it is very clear that in the manganese perovskites, there is a very large driving force toward the equilibrium segration of calcium: values of $-50.3 \mathrm{~kJ} / \mathrm{mol}$ for the heat of calcium segregation have been measured in magnesium oxide. ${ }^{7}$ When there is little significant segregation the surface is likely to be unstable toward surface reconstruction or surface relaxation. These differences at the surface suggest that under equilibrium conditions, the surface will not have a composition and/or structure representative of the bulk.

Our results provide strong indications of a large difference between the surface free enthalpy and the bulk, with the consequence of another fundamental conclusion. If by adopting some cleaning procedure whereby one had a surface terminal layer with the stoichiometry and structure of the bulk, then the surface will be at a free enthalpy not representative of the bulk. This latter condition would mean that the electronic structure at the surface would not be representative of the bulk. This last complexity casts great doubt on the premise that photoemission, a surface sensitive technique, can be used to determine successfully the bulk electronic structure of these complex oxides. Nor can one assume that the electronic structure of the surface, at equilibrium segregation, is representative of a bulk sample of similar composition.

The existance of strong differences in the surface enthalpy from the bulk can have a profound influence on the surface electronic structure. In the absence of surface segregation (the nonequilibrium condition) we would expect strong con- tributions from surface states and resonances. This is in fact what we and others ${ }^{21}$ observe for complex oxides.

In manganese perovskites systems with surface resonances, the bulk phase transition has been correlated to changes observed in conventional photoemission. ${ }^{1,18,22}$ As is clear from the above discussion, this still does not necessarily imply that the surface electronic structure is representative of the bulk. ${ }^{1}$ Angle resolved photoemission measurements need to be undertaken at different photoenergies with some efforts made to distinguish between surface and bulk contributions of the observed electronic structure in photoemission.

\section{CONCLUSION}

These results strongly indicate that before any angle resolved photoemission study is undertaken for complex metal oxides, angle resolved XPS studies like this one, or some equivalent measurement, should be undertaken. In the absence of surface segregation, samples of the complex oxides should be studied carefully to ascertain the surface contributions to the electronic structure.

\section{ACKNOWLEDGMENTS}

This work was funded by the National Science Foundation (NSF) through Grant Nos. DMR-92-21655/94-96131 and INT-93-00238 and the Joint Research Center for Atomic Technologies. This work was carried out, in part, at the Synchrotron Radiation Center in Stoughton, Wisconsin, which is funded by the NSF. The authors would like to thank Jiandi Zhang, E. W. Plummer and David McIlroy for their assistance and a number of useful remarks.

Presented at the 44th National Symposium of the American Vacuum Society, San Jose, CA, 20-24 October 1997.

${ }^{1}$ D. N. McIlroy, C. Waldfried, J. Choi, F. Foong, S.-H. Liou, and P. A. Dowben, Phys. Rev. B 54, 17438 (1996).

${ }^{2}$ C. Waldfried, D. N. McIlroy, S.-H. Liou, R. Sabiryanov, S. S. Jaswal, and P. A. Dowben, J. Phys.: Condens. Matter 9, 1031 (1997).

${ }^{3}$ S. H. Liu and R. A. Klemm, Phys. Rev. Lett. 73, 1019 (1994).

${ }^{4}$ M. Lindroos and A. Bansil, Phys. Rev. Lett. 75, 1182 (1995).

${ }^{5}$ E. A. Colbourn, W. C. Mackrodt, and P. W. Tasker, J. Mater. Sci. 18, 1917 (1983).

${ }^{6}$ W. C. Mackrod and P. W. Tasker, J. Am. Ceram. Soc. 72, 1576 (1989).

${ }^{7}$ R. C. McCune and P. Wynblant, J. Am. Ceram. Soc. 66, 111 (1983).

${ }^{8}$ J. Choi, S.-H. Liou, P. A. Dowben, J. Zhang, and E. W. Plummer (submitted).

${ }^{9}$ J. Choi, S.-H. Liou, P. A. Dowben, and M. Langell (unpublished).

${ }^{10}$ J. H. Scofield, J. Electron Spectrosc. Relat. Phenom. 8, 129 (1976).

${ }^{11}$ Surface Segregation Phenomena, edited by P. A. Dowben and A. Miller (CRC, Boston, MA, 1990), p. 145.

${ }^{12}$ U. Vahalia, P. Dowben, and A. Miller, J. Vac. Sci. Technol. A 4, 1675 (1986).

${ }^{13}$ U. Vahalia, P. Dowben, and A. Miller, J. Electron Spectrosc. Relat. Phenom. 37, 303 (1986).

${ }^{14}$ D. R. Penn, J. Electron Spectrosc. Relat. Phenom. 9, 29 (1976).

${ }^{15}$ G. Frank, Ch. Ziegler, and W. Gøpel, Phys. Rev. B 43, 2828 (1991); N. Terada, S. Ishibashi, M. Jo, M. Hirabayashi, H. Ihara, and S. Yamamoto, Appl. Phys. Lett. 63, 2967 (1993).

${ }^{16}$ L.-C. Dufour, G. L. Bertrand, G. Caboche, P. Decorse, A. El Anesari, A. Poirson, and M. Vareille, Solid State Ionics 101-103, 661 (1997).

${ }^{17}$ S. Y. Liu and H. H. Kung, Surf. Sci. 110, 504 (1981). 
${ }^{18}$ J. H. Park, C. T. Chen, S.-W. Cheong, W. Bao, G. Meigs, V. Chakarian, and Y. U. Idzerda, Phys. Rev. Lett. 76, 4215 (1996); A. Chaiani, H. Kumigashira, T. Takashi, Y. Tomioka, H. Kuwahara, and Y. Torkura (submitted).

${ }^{19}$ A. Biswas, A. K. Raychaudhuri, R. Mahendiran, A. Guha, R. Mahesh, and C. N. R. Rao, J. Phys.: Condens. Matter 9, L355 (1997).
${ }^{20}$ T. Yokoya, A. Chainani, T. Takahashi, H. Ding, J. C. Campuzano, H. Katayama-Yoshida, M. Kasai, and Y. Tokura, Phys. Rev. B 54, 13311 (1996); A. P. Mackenzie et al., Phys. Rev. Lett. 76, 3786 (1996).

${ }^{21}$ A. A. Zakharov, H. Nylén, M. Quarford, I. Lindau, M. Leandersson, M. B. Tsetlin, and M. N. Mikheeva, Phys. Rev. B 56, 9030 (1997).

${ }^{22}$ D. N. McIlroy, J. Zhang, S.-H. Liou, and P. A. Dowben, Phys. Lett. A 207, 367 (1995). 